\title{
VOLTAGE DETECTION AND MAGNET PROTECTION
}

\author{
J.M. Pfotenhauer, F. Kessler, and M.A. Hilal \\ Applied Superconductivity Center \\ University of Wisconsin-Madison \\ 1500 Johnson Drive \\ Madison, WI 53706
}

Abstract - Voltage detection is routinely used to identify resistive regions within superconducting magnets so that a protection circuit can trigger a safe magnet discharge. Non-resistive voltage signals, for example inductive voltages, can however produce false signals causing the magnet protection system to trip prematurely. An experimental verification is given of a quench detection method which eliminates sensitivity to inductive voltages in multiple magnet systems. In addition, a specific application of these ideas for the UW Proof of Principle Experiment demonstrates the need for incorporating a microprocessor in the quench detection system.

\section{INTRODUCTION}

Magnet protection mandates the immediate detection of any normal zone so that the magnet can be discharged. The detection circuit should respond to small normal zone resistive voltages but should not respond to other voltage spikes or fluctuations which may be induced by other sources such as power supplies. For the traditional method of quench detection the magnet is usually provided with three voltage taps. One of the voltage taps is typically located at the center of the windings and the voltages across the two halves of the magnet are compared and balanced so that they are equal. A normal zone will induce an imbalance voltage and can thus be detected. The above technique has been successfully used for is single windings but it has shown by Hilal et $\mathrm{al}^{(1)}$ that such a technique is not adequate to use for magnet protection of multiple windings. In that report the theoretical foundation is given for a technique which eliminates sensitivity in the quench detection system to any induced voltages resultant from mutual or self inductances of the various coupled magnets. The quench detector then sees only resistive voltages resultant from normal zones in the coil. The formalism introduced by Hilal et al calculates appropriate gain values, $G$, for the amplifier components of the quench detection circuits based on the inductive coupling co-efficients $a_{i j k}$ of the ith magnet, $j$ th section, with the kth magnet. We present in this paper the experimental verification of the new technique and how it will be applied to the Proof of Principle Experiment (POPE) facility constructed at the University of Wisconsin. The facility is a high current test facility which is presently being used for the SMES conductor test. A detailed description of the facility is given elsewhere ${ }^{(2)}$ but a brief description is given below.

Acknowledgements: Work partially funded through the Navy and DNA. Manuscript received August 24, 1992.

\section{EXPERIMENT}

The following experimental results verify the quench detection method of Hilal et a ${ }^{(1)}$. In addition, shortcomings of the traditional quench detection techniques are demonstrated explicitly.

\section{A. Equipment}

Two superconducting solenoids are arranged on the same axis, but with vertical centers offset (see Fig. 1).

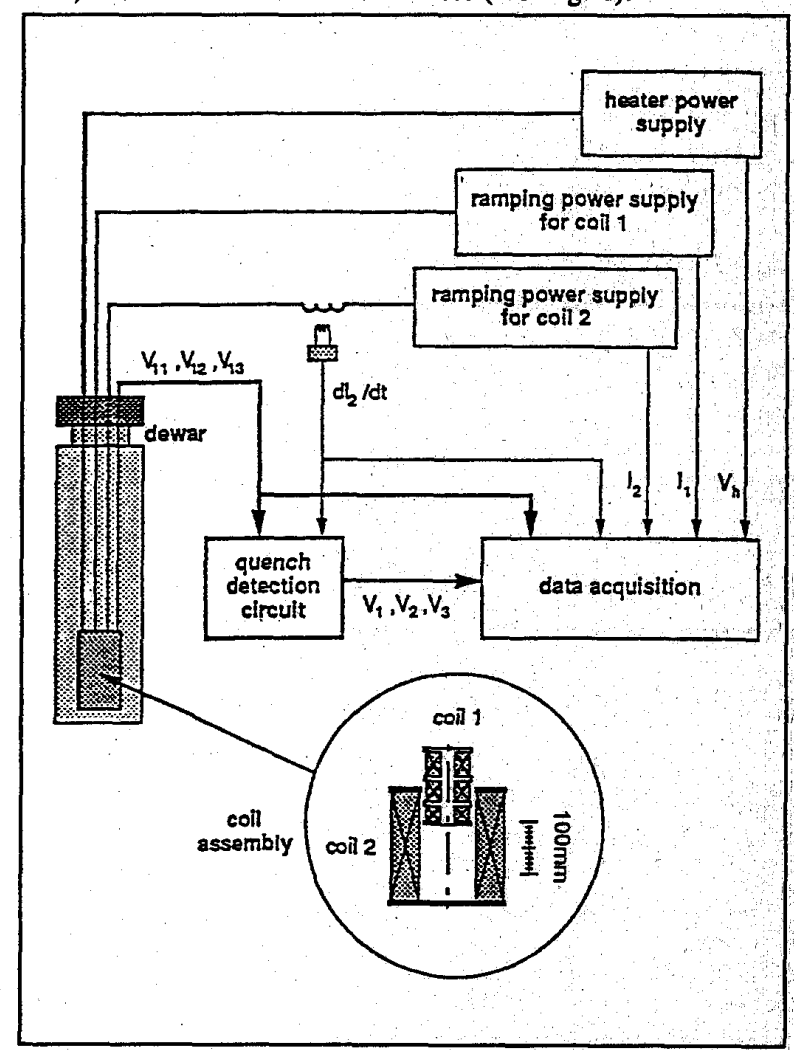

Fig. 1 Experimental Set-up

As prescribed by the analysis the coil being monitored for normal zones is divided into three sections. In this case the three sections are vertically displaced from each other, but all are wound from a continuous length of superconducting wire of $0.508 \mathrm{~mm}$ diameter and with a copper to superconducting ratio of 1.7. The measured inductances, both self and mutual, 
of the two coils are listed in Tab. 1.

Table 1: Coil and Current Parameters

\begin{tabular}{|c|c|c|c|}
\hline \multicolumn{2}{|c|}{ self inductances } & \multicolumn{2}{|c|}{ mutual inductances } \\
\hline$a_{111}$ & $0.344 \mathrm{H}$ & $a_{112}$ & $0.136 \mathrm{H}$ \\
\hline$a_{121}$ & $0.291 \mathrm{H}$ & $a_{122}$ & $0.354 \mathrm{H}$ \\
\hline$a_{131}$ & $0.200 \mathrm{H}$ & $a_{132}$ & $0.492 \mathrm{H}$ \\
\hline$a_{212}$ & $19.8 \mathrm{H}$ & \multirow{2}{*}{\multicolumn{2}{|c|}{ max. ramping rates }} \\
\hline \multicolumn{2}{|c|}{ max. currents } & & \\
\hline$i_{1}$ & $11 \mathrm{~A}$ & $\mathrm{dl}_{1} / \mathrm{dt}$ & $0.4 \mathrm{~A} / \mathrm{s}$ \\
\hline $\mathrm{I}_{2}$ & $20 A$ & $\mathrm{dl}_{2} / \mathrm{dt}$ & $0.2 \mathrm{~A} / \mathrm{s}$ \\
\hline
\end{tabular}

Separate and independent power supplies are used to ramp the current in each coil with maximum values of $\mathrm{I}_{1}, \mathrm{I}_{2}, \mathrm{dI}_{\mathrm{L}} / \mathrm{dt}$, and $\mathrm{dL}_{2} / \mathrm{dt}$ as given also in Tab. 1. Critical currents of the two magnets are at least an order of magnitude larger than the values used in this experiment. Voltage tap pairs are installed on coil 1 for measuring the voltages across each of the three sections. In addition, two heaters installed on coil 1 provide a means of generating a normal zone in one or more of the coil sections. Current ramping rates can be predetermined at the power supplies and an additional inductive pick-up is incorporated on the room temperature section of the coil 2 charging circuit for measuring $\mathrm{dL}_{2} / \mathrm{dt}$. In all of the tests, the superconducting coils are operated in a saturated bath of liquid helium at $4.2 \mathrm{~K}$.

Fig. 2 displays the different circuits used for detecting a normal zone in coil 1 . As will be discussed below, the first of

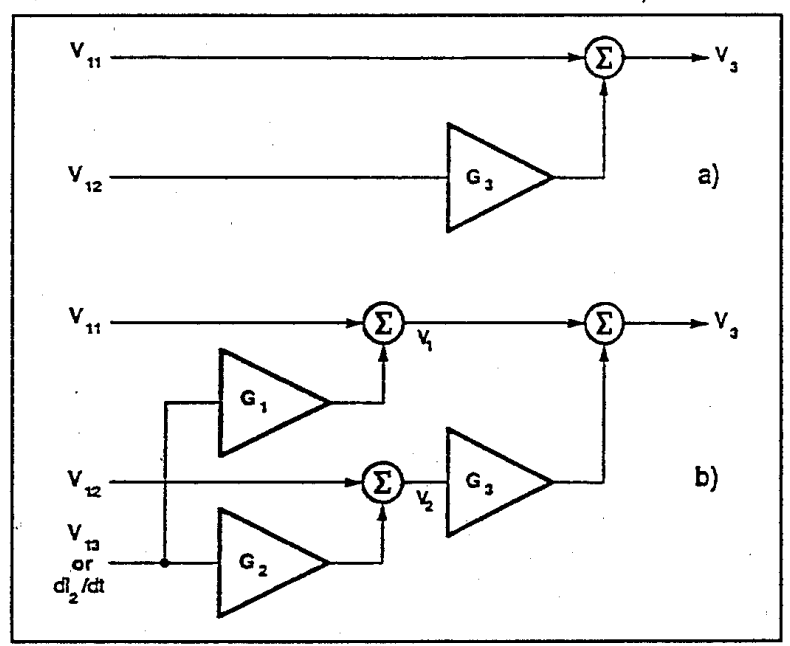

Fig. 2 Quench detection circuits

a) traditional quench detection method

b) gaussion elimination method these circuits is vulnerable to false quench signals while the other will eliminate that problem. The circuit shown in Fig. 2a is appropriate for the standard technique using a two-pairedvoltage-tap measurement. Fig. $2 b$ displays the circuit appropriate for the "gaussian elimination" technique in a two magnet system in which the monitored coil (coil 1) is instrumented with three voltage tap pairs. The alternate circuit choice of Fig. 2b allows using a two-paired-voltage-tap detection system but requires measurement of $\mathrm{dl}_{2} / \mathrm{dt}$ from the other coil(s). In each of these circuits the signals from the voltage tap pairs are input at the left and the quench detection signal is output at the far right. Adjustable gain amplifiers are denoted by the letter "G".

\section{B. Procedure and results}

The experimental activities are divided into three groups corresponding to the various quench detection circuits of Fig. 2. In the first test adjacent voltage tap pairs across the top two sections of coil 1 are used to provide input signals for the quench detector circuit shown in Fig. 2a. This configuration simulates the standard technique of monitoring voltages in two halves of a magnet by using a common "center tap" in each of the voltage tap pairs. In keeping with the traditional technique, we measured the two voltages $V_{11}$ and $V_{12}$ while ramping the current in coil $1 . G_{3}$ is adjusted during the ramping procedure until $V_{3}=0$. In this condition $G_{3}$ compensates for the different self inductances of the two sections of the coil and according to the theory has the value given by

$$
G_{3}=a_{111} / a_{121}
$$

Fig. 3 displays the output of $V_{3}$ after $G_{3}$ has been set and

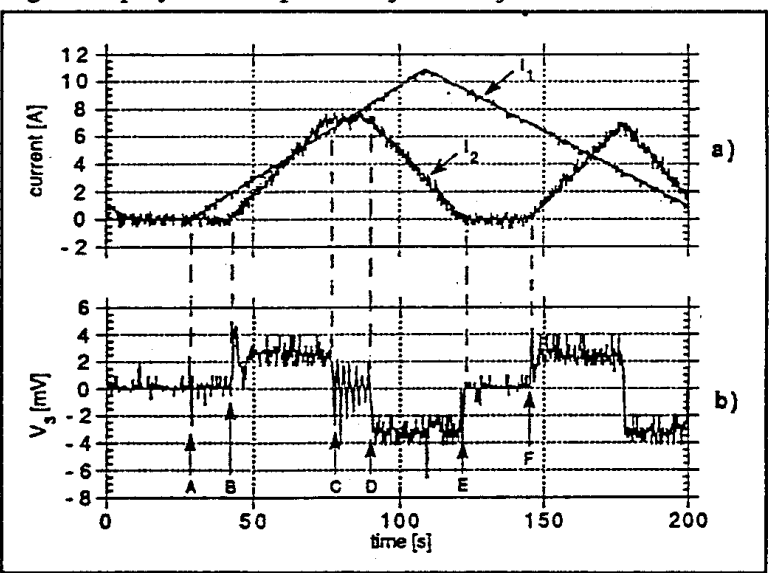

Fig. 3 Traditional quench detection $G_{3}$ adjusted for inductance ratio

demonstrates that as long as $\mathrm{dL}_{2} / \mathrm{dt}=0$ the traditional quench detection method eliminates inductive voltage signals from the output of the quench detector circuit; see especially segment AB. However, whenever $\mathrm{dL}_{2} / \mathrm{dt} \neq 0$ a non-zero voltage signal appears at $V_{3}$ and can be erroneously identified as a normal 
zone voltage. Note that in the very unusual chance that the mutual inductance ratio of coil 1 equals the self inductance ratio; that is

$$
a_{112} / a_{122}=a_{111} / a_{121}
$$

the traditional quench detection method would also eliminate induced voltages from coil 2. An additional aspect of the first test is shown in Fig. 4. Here, prior to point $A$ it is demonstrated that $\mathrm{G}_{3}$ can be adjusted for fixed ramping rates of coil 1 and coil 2 , such that $V_{3}=0$ and all inductive voltage signals are eliminated. This technique breaks down however as soon as there is any deviation from the specific ratio of $\mathrm{I}_{1} / \mathrm{I}_{2}$ for which the setting of $G_{3}$ produces a zero voltage output at $V_{3}$.

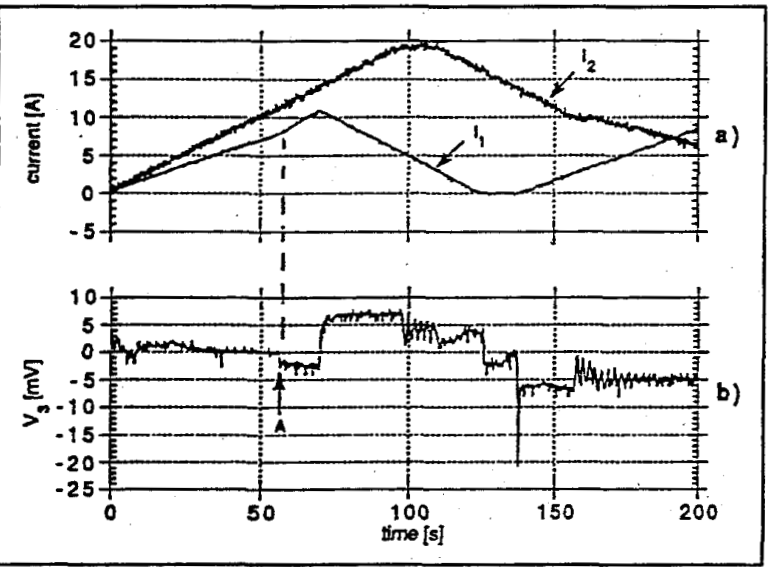

Fig. 4 Traditional quench detection $G_{3}$ adjusted for fixed current ramping ratio of both magnets

In the second test the three adjacent voltage tap pairs encompassing coil 1 provide the input for the quench detection circuit shown in Fig. 2b. The "gaussian elimination" circuit is so named after the corresponding matrix procedure for solving a set of $\mathbf{n}$ equations with $\mathbf{n}$ unknowns. In the equivalent circuit adjustment procedure, specific values for $G_{1}, G_{2}$, and $G_{3}$ are determined so that $V_{3}=0$ for all values (and combinations) of $\mathrm{dL}_{1} / \mathrm{dt}$ and $\mathrm{dI}_{2} / \mathrm{dt}$. Any non-zero voltage appearing at $\mathrm{V}_{3}$ is then correctly identified as a resistive voltage. In the first step in the adjustment procedure, we ramp $I_{1}$ alone and adjust $G_{1}$ and $G_{2}$ so that $V_{1}=0$ and $V_{2}=0$. From the theoretical considerations these are $G_{1}=-a_{11} / a_{131}$ and $G_{2}=-a_{121} / a_{131}$. This step reduces the number of unknowns from three to two since sensitivity to $\mathrm{dL}_{\mathrm{L}} / \mathrm{dt}$ is eliminated. The second step is accomplished by ramping $I_{2}$ and adjusting $G_{3}$ so that $V_{3}=0$. In this step voltages appearing at $V_{1}$ and $V_{2}$ due to the different mutual inductances $a_{112}, a_{12}$, and $a_{132}$ are compensated by $G_{3}$. From the theoretical considerations $G_{3}$ is given by

$$
G_{3}=-\frac{a_{112} a_{131}-a_{111} a_{132}}{a_{122} a_{131}-a_{121} a_{132}}
$$

The results from the experimental test of this procedure are shown in Fig. 5. The outputs of $V_{1}$ and $V_{2}$, shown in Fig. $5 b$ and $5 \mathrm{c}$, display no sensitivity to changes in $\mathrm{dL}_{1} / \mathrm{dt}$ and are zero

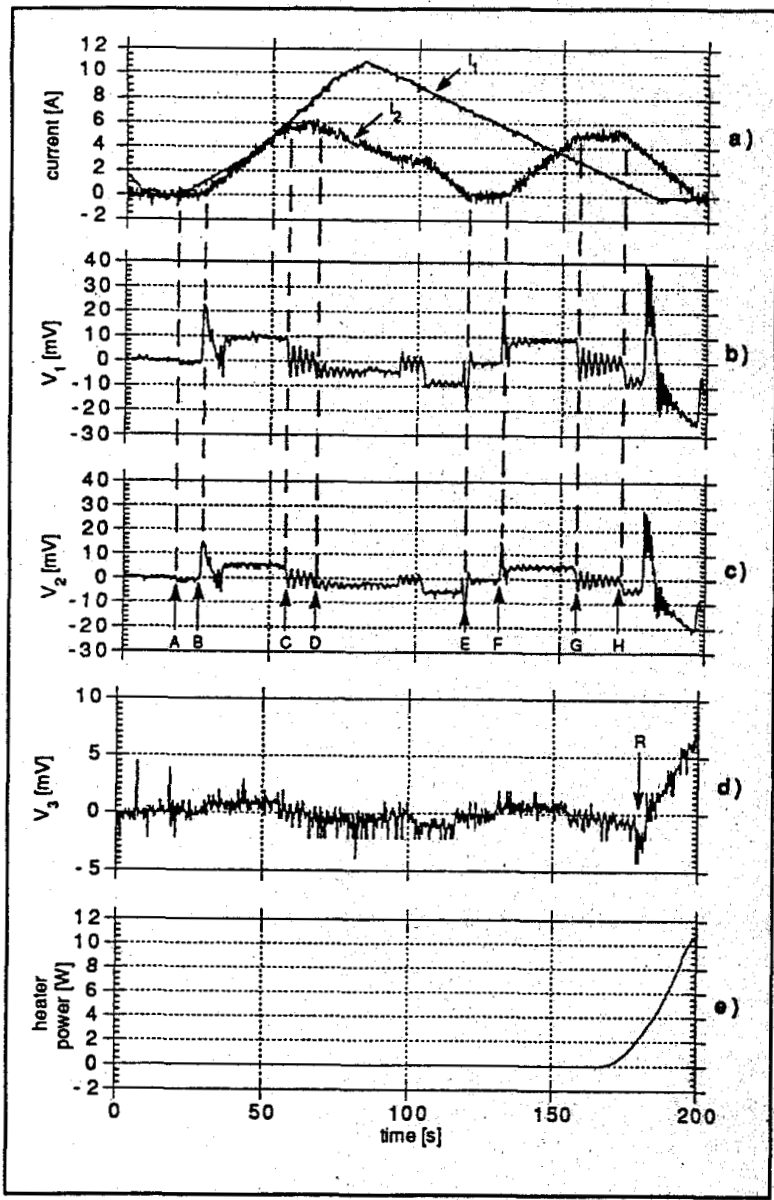

Fig. 5 Current and voltage measurements using gaussion elimination technique see test for details

when $\mathrm{dl}_{2} / \mathrm{dt}=0$, see segments $\mathrm{A}-\mathrm{B}, \mathrm{C}-\mathrm{D}, \mathrm{E}-\mathrm{F}, \mathrm{G}-\mathrm{H}$. Note that the different magnitudes of $V_{1}$ and $V_{2}$ are exactly compensated by $G_{3}$ so that $V_{3}=0$ to within $\pm 2.5 \mathrm{mV}$. Fig. 5e displays the power dissipated in a manganin wire heater which is wound around a $17 \mathrm{~cm}$ length of the coil 1 superconductor. That same section of the heater and superconductor is covered by epoxy to minimize cooling over that length. The resistive normal zone generated by these conditions shows up clearly at the output of $V_{3}$ as seen to the right of point $R$ on Fig. $5 d$. Note that this detection scheme is able to identify a resistive voltage that is 20 times smaller than inductive voltages present at the $V_{11}, V_{12}$, and $V_{13}$ inputs. At this point we have not yet defined the maximum amount of inductive voltage which can be eliminated while maintaining this sensitivity, but it is likely that it can do even better than the results shown here.

In the third and final test, the quench detection circuit is the same as that shown in Fig. $2 b$ with the exception that $V_{13}$ is replaced by a signal proportional to $\mathrm{dL}_{2} / \mathrm{dt}$ An inductive 
pick-up coil coupled to the room temperature portion of the

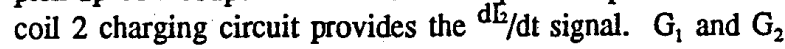
perform the function of compensating for the voltages induced to sections 1 and 2 respectively of coil 1 due to $\mathrm{dL}_{2} / \mathrm{dt} . \mathrm{G}_{3}$ compensates for the difference in the self inductances of sections 1 and 2 of coil 1 thereby eliminating sensitivity to $\mathrm{dI}_{1} / \mathrm{dt}$. As shown in Fig. 6 the output voltage at $\mathrm{V}_{3}$ is insensitive to $\mathrm{dI}_{1} / \mathrm{dt}$ and $\mathrm{dI}_{2} / \mathrm{dt}$

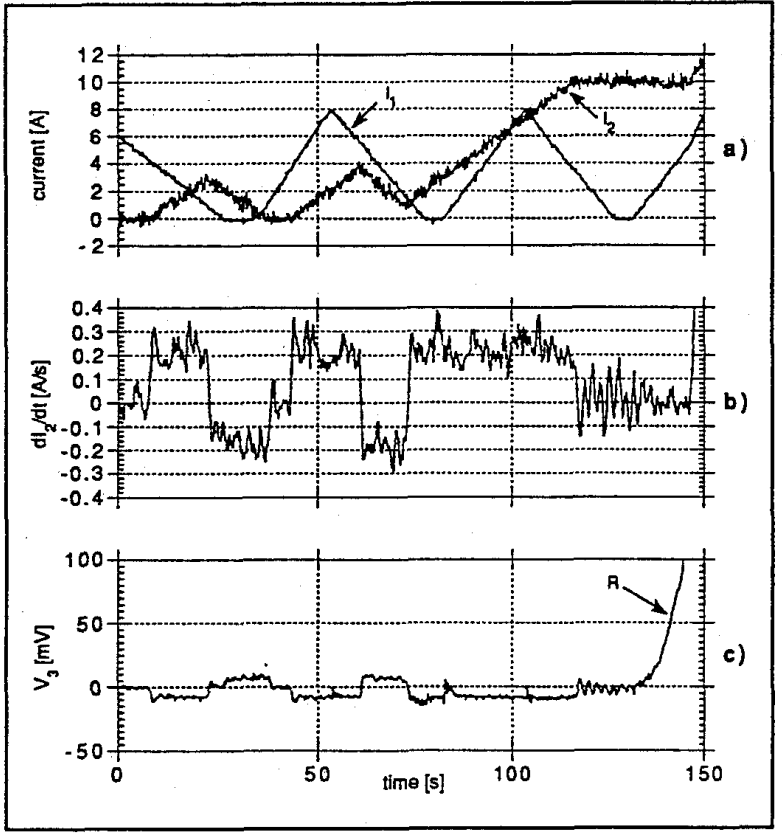

Fig. 6 Gaussian elimination technique using $\mathrm{dl}_{2} / \mathrm{dt}$ at third input

but displays the resistive voltage which corresponds to that generated by the manganin heater. Note that this quench detection method is preferred for a magnet system where the number of coupled magnets may change. Traditional twopaired-voltage-tap detection systems can be augmented by signals proportional to ${ }^{\mathrm{dL}} / \mathrm{dt}$ for each ith magnet added to the system.

\section{APPLICATION TO POPE}

The charging circuits and magnet configurations for the UW-Madison Proof of Principle Experiment are shown in Fig. 7. Operation of the POPE requires that the background magnet quench detection system be insensitive to any current fluctuations in the test coil. However, the mutual coupling between the test coil and the background coils is strong due to their close proximity. Therefore, since the existing voltage tap arrangement for the background coil is, a two-paired-voltagetap configuration (see Fig. 7), it requires the additional information of $\mathrm{dr} / \mathrm{dt}$ from the test coil for a workable quench detection system.

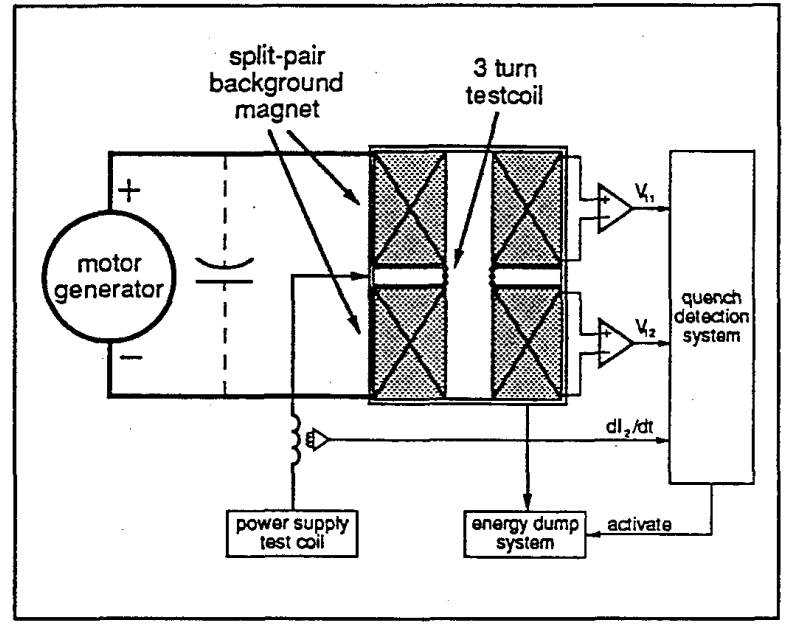

Fig. 7 Circuit configuration for test coil, background coil, and quench detection system in the POPE

A further complication for the quench detector system has been identified in the induced voltage signals $V_{11}$ and $V_{12}$ of the background coils. Specifically, voltages recorded at $V_{11}$ and $V_{12}$ during ramp rates of the test coil which are slower than $350 \mathrm{amps} / \mathrm{sec}$ are nearly equal and of the same size. However, voltages recorded in $\mathrm{V}_{11}$ and $\mathrm{V}_{12}$ in response to test coil fluctuations of $1200 \mathrm{amps} / \mathrm{sec}$ or faster are nearly equal but of opposite sign. This behavior is attributed to a virtual capacitance of the motor generator. Thus, for transient induced voltages in the background coil, the motor generator acts as a short across the background coils. The induced voltage profile existent in a superconducting magnet which is shorted across its end terminals has been described by Hilal ${ }^{(3)}$, and when combined with the fact that the center voltage tap is unlikely to be at the exact inductive center of the background magnet, provides a reasonable explanation of the equal and opposite voltages observed. To accommodate this added complication, the quench detector system for the POPE is equipped with a micro-processor to make real time identification and elimination of both types of voltages induced in the background coil from charges in the test coil current.

\section{REFERENCES}

[1] M.A. Hilal, J.M. Pfotenhauer, F. Kessler, and G. Veccey, "Quench Detection of Multiple Magnet System," submitted to Cryogenics 9/92.

[2] J.M. Pfotenhauer, F. Bodker, Z. Jiang, O.D. Lokken, B. Tao, D. Yu, g.E. McIntosh, and L.E. Nickels, "A 100kA, HeII Cooled Conductor Test Facility," in Advances in Cryogenic Engineering, R.W.Fast ed. vol 37A Plenum, New York, June 1991, pp 155-162.

[3] M.A. Hilal, "Internal Voltage Distribution in Magnet Systems,", IEEE Trans. on Magnetics vol Mag-19(3), May 1983, pp 1101. 